\title{
Essential Supremum and Essential Maximum with Respect to Random Preference Relations
}

\author{
Yuri KABANOV ${ }^{\mathrm{a}}$, Emmanuel LEPINETTE ${ }^{\mathrm{b}}$ \\ ${ }^{a}$ University of Franche Comté, Laboratoire de Mathématiques, 16 Route de Gray, \\ 25030 Besançon cedex, France, and \\ National Research University - Higher School of Economics, Laboratory of Quantitative \\ Finance, Moscow, Russia. Email: youri.kabanov@univ-fcomte.fr \\ ${ }^{b}$ Paris-Dauphine University, Ceremade, Place du Maréchal De Lattre De Tassigny, \\ 75775 Paris cedex 16, France. Email: emmanuel.lepinette@ceremade.dauphine.fr.
}

\begin{abstract}
In the first part of the paper we study concepts of supremum and maximum as subsets of a topological space $X$ endowed by preference relations. Several rather general existence theorems are obtained for the case where the preferences are defined by countable semicontinuous multi-utility representations. In the second part of the paper we consider partial orders and preference relations "lifted" from a metric separable space $X$ endowed by a random preference relation to the space $L^{0}(X)$ of $X$-valued random variables. We provide an example of application of the notion of essential maximum to the problem of the minimal portfolio super-replicating an American-type contingent claim under transaction costs.
\end{abstract}

Keywords: Preference Relation, Partial Order, Random cones, Transaction costs, American option, Hedging.

2000 MSC: 60G44, G11-G13.

\section{Introduction}

This paper pursues several purposes.

First, we continue to study the concepts of supremum and essential supremums as sets, initiated in [8] and where the reader can find a detailed motivation of interest to these and related objects. In contrast with the mentioned paper where we worked in the "elementary" framework of $\mathbf{R}^{d}$ with a partial 
order given by a countable continuous multi-utility representation, we consider here much more general one, namely, of a topological space $X$ equipped with a preference relation (preorder) and obtain, in this standard setting of the modern preference theory, extensions of several results from [8].

Our interest to such a general setting is motivated, essentially, by the models of financial markets with transaction cost with infinite many (and even uncountably many securities), see the recent works [4] and [3].

Second, we introduce, for a set $\Gamma \subseteq X$, a concept of Max $\Gamma$ as a subset of the closure $\bar{\Gamma}$ and provide some characterizations of this set together with sufficient conditions ensuring that it is not empty.

Third, and this is our main purpose, we study the concepts of essential supremum and essential maximum in the space $L^{0}(X)$ of random variables taking values in a separable metric space $X$, assuming that $L^{0}(X)$ is equipped with a preference relation induced by a (possibly, random) preference relation in $X$. These objects of interest are subsets of $L^{0}(X)$. Special attention is payed to the case where $X$ is a separable Hilbert space and the preorder is given by a random cone.

Forth, we apply the abstract theory to describe the set of minimal hedging portfolios in the problem of hedging of American-type contingent claims in the presence of proportional transaction costs.

In the theory of markets with friction which can be found in the book [9] the value processes are $d$-dimensional adapted processes and so are American contingent claims. Hedging (super-replicating) a contingent claim $Y=\left(Y_{t}\right)$ means to find a value process $V=\left(V_{t}\right)$ which dominates the claim in the sense that for any $t$ the difference $V_{t}-Y_{t}$ belongs to the solvency cone $K_{t}$, i.e. $V_{t}$ dominates $Y_{t}$ in the sense of the preference relation generated by the cone $K_{t}$ (the latter is a partial order if $K_{t}$ is proper, as assumed in models with "efficient friction"). The solvency cone is a fundamental notion of the theory giving a geometric description of the vectors of investor's positions that can be converted (paying the transaction costs) into vectors with non-negative components. In general, solvency may depend on the state of the nature $\omega$ and this is always the case when models are described in physical units and the latter description is more convenient for analysis.

Though in the existing models of financial markets $K_{t}(\omega)$ are polyhedral cones, mathematically it is quite reasonable to consider more abstract models where $K=\left(K_{t}\right)$ is an adapted set-valued process which values are closed convex cones, see [14].

The value process $V$ is called minimal if it dominates $Y$, at the terminal 
date $V_{T}=Y_{T}$, and any value process $W$ dominating $Y$ and dominated by $V$ dominates $V$. The problem of interest is whether the minimal portfolios do exist and how they can found. We provide a description of the set of minimal portfolios as the solution of (backward) recursive inclusions. In a striking contrast with the description obtained in [8] for European claims and involving Esssup, the present one, for the American options, is based on the concept Essmax. The developed theory covering preorders allows us to work without the efficient friction condition.

The structure of the paper is the following. In Section 2 we consider a quite general deterministic setting when the preference relation on a topological space is given by a family of semicontinuous functions. We extend our previous results on the existence of $\operatorname{Sup} \Gamma$ (as a no-empty set). We define the set Max $\Gamma$ and give sufficient conditions to guarantee its existence. In the preference theory and multivariate optimization there is a plethora of definitions of supremum/maximum-like objects, see, e.g. [5], [11], [15], but we could identify ours with already known and the approach based on multiutility representation seems to be new. In Section 3 we work in a setting where $X$ is a separable metric space and the preference relation in the space of $X$-valued random variables is given by a countable family of Carathéodory functions. We discuss results on the existence of Esssup $\Gamma$ and Essmax $\Gamma$ for an order bounded set of $X$-valued random variables. In Section 4 we consider a more specific case where the random preference relation is given by a random cone. In Section 5 we give an application to the hedging problem for American-type contingent claims under transaction costs.

\section{Supremum and Maximum with Respect to a Preference Rela- tion in a Deterministic Setting}

\subsection{Basic Concepts}

Let $\succeq$ be a preference relation or a preorder in $X$, i.e. a binary relation between certain elements of a set $X$ which is reflexive $(x \succeq x)$ and transitive (if $x \succeq y$ and $y \succeq z$ then $x \succeq z$ ). The preorder is called partial order if it is antisymmetric (if $x \succeq y$ and $y \succeq x$ then $x=y$ ).

Define an order interval $[x, y]:=\{z \in X: y \succeq z \succeq x\}$ and extend this notation by putting

$$
]-\infty, x]:=\{z \in X: x \succeq z\}, \quad[x, \infty[:=\{z \in X: z \succeq x\}
$$


(the latter objects are also called lower and upper contour sets). If $\Gamma$ is a subset of $X$, the notation $\Gamma \succeq x$ means that $y \succeq x$ for all $y \in \Gamma$. In the same spirit: $\Gamma_{1} \succeq \Gamma$ means that $x \succeq y$ for all $x \in \Gamma_{1}$ and $y \in \Gamma$;

$$
\left[\Gamma, \infty\left[:=\cap_{z \in \Gamma}\{z \in X: z \succeq x\}\right.\right.
$$

etc. We shall use the notation $x \preceq z$ instead of $z \succeq x$.

For the case where $X$ is a topological space (this will be the standing assumption in this paper), the following definitions are used: a preference relation is upper semi-continuous (respectively, lower semi-continuous) if $[x, \infty[$ (respectively, $]-\infty, x]$ ) is closed for any $x \in X$ and semi-continuous if it is both upper and lower semi-continuous. It is called continuous if its graph $\{(x, y): y \succeq x\}$ is a closed subset of $X \times X$.

Let $Y$ be a set equipped by a preference relation $\succeq^{Y}$. We say that a set $\mathcal{U}$ of $Y$-valued functions defined on $X$ represents the preference relation $\succeq$ if for any $x, y \in X$,

$$
x \succeq y \Leftrightarrow u(x) \succeq^{Y} u(y) \quad \forall u \in \mathcal{U}
$$

In the literature usually $Y=\mathbf{R}$, i.e. $\mathcal{U}$ is a set of real functions on $X$ and

$$
x \succeq y \Leftrightarrow u(x) \geq u(y) \quad \forall u \in \mathcal{U}
$$

In the sequel of the paper, by default, $Y=\mathbf{R}$ if not specified particularly.

This set $\mathcal{U}$ is called multi-utility representation of the preference relation. If its elements are (semi)continuous functions, we say that $\mathcal{U}$ is a (semi)continuous multi-utility representation of the preference relation.

Clearly, any preference relation can be represented by the family of indicator functions $\mathcal{U}:=\left\{I_{[x, \infty[}, x \in X\right\}$.

The following statements (between many other interesting results) are due to Evren and Ok, [7]:

1) any upper (lower) semicontinuous preference relation on a topological space $X$ admits an upper (lower) semicontinuous multi-utility representation,

2) any continuous preference relation on a locally compact $\sigma$-compact topological space $\mathrm{X}$ admits a continuous multi-utility representation.

Note that an arbitrary family $\mathcal{U}$ defines a preference relation. It is a partial order if the equalities $u(x)=u(y)$ for all $u \in \mathcal{U}$ imply that $x=y$.

The elements $x$ and $y$ are equivalent if $x \succeq y$ and $y \succeq x$; we write $x \sim y$ in this case. 


\subsection{Supremum as a Set}

Definition 2.1. Let $\Gamma$ be a non-empty subset of $X$ and let $\succeq$ be a preference relation. We denote by $\operatorname{Sup} \Gamma$ the largest subset $\hat{\Gamma}$ of $X$ such that the following conditions hold:

$\left(a_{0}\right) \hat{\Gamma} \succeq \Gamma$;

$\left(b_{0}\right)$ if $x \succeq \Gamma$, then there is $\hat{x} \in \hat{\Gamma}$ such that $x \succeq \hat{x}$;

$\left(c_{0}\right)$ if $\hat{x}_{1}, \hat{x}_{2} \in \hat{\Gamma}$, then $\hat{x}_{1} \succeq \hat{x}_{2}$ implies $\hat{x}_{1} \sim \hat{x}_{2}$.

Remark 2.2. If the relation $\succeq$ is a partial order, the equivalence $\hat{x}_{1} \sim \hat{x}_{2}$ means simply that $\hat{x}_{1}=\hat{x}_{2}$. In the case of partial order the set $\hat{\Gamma}$ satisfying $\left(a_{0}\right),\left(b_{0}\right),\left(c_{0}\right)$ is unique, see Lemma 3.3 in [8], but, for a general preference relation this is not true. Note that $\operatorname{Sup} \Gamma$ may not exist. It is easy to see that $\operatorname{Sup} \Gamma$ is the union of all subset $\hat{\Gamma}$ satisfying $\left(a_{0}\right),\left(b_{0}\right),\left(c_{0}\right)$.

The equivalence relation $\sim$ defines the quotient space $\tilde{X}:=X / \sim$; the notation $[x]$ means the class containing an element $x \in \tilde{X}$. The relation $[y] \succeq[x]$ is a partial order in $\tilde{X}$. Let us consider the weakest topology in $\tilde{X}$ under which the quotient mapping $q: x \mapsto[x]$ is continuous.

Note that the continuity of $q$ is equivalent to the inclusion $q(\bar{\Gamma}) \subseteq \overline{q(\Gamma)}$ for any subset $\Gamma$ of $X$ ( $\bar{\Gamma}$ denotes the closure of $\Gamma)$, see [10], Ch. 3, Th. 1 . Since $q(\Gamma) \subseteq q(\bar{\Gamma})$ we have the equality $\overline{q(\Gamma)}=\overline{q(\bar{\Gamma})}$.

We summarize in the following lemma several obvious properties.

Lemma 2.3. Let $\Gamma \subseteq X$. Then

(i) $\operatorname{Sup} \Gamma=\operatorname{Sup} \Gamma^{\sim}$, where

$$
\Gamma^{\sim}:=\left\{x^{\prime}: \text { there exists } x \in \Gamma \text { such that } x^{\prime} \sim x\right\}=q^{-1} q(\Gamma) ;
$$

(ii) if $\widehat{\Gamma}=\operatorname{Sup} \Gamma$, then $q(\widehat{\Gamma})=\operatorname{Sup} q(\Gamma)$;

(iii) if $A \subseteq \tilde{X}$ and $\operatorname{Sup} A$ exists, then $q^{-1}(\operatorname{Sup} A)=\operatorname{Sup} q^{-1}(A)$.

If the set $\widehat{\Gamma}=\operatorname{Sup} \Gamma$ for $\Gamma \subseteq X$, then its image $q(\widehat{\Gamma})$ coincides with $\operatorname{Sup} q(\Gamma)$ in the quotient space $\tilde{X}$. Also, $\widehat{\Gamma}=\operatorname{Sup} q^{-1}(q(\Gamma))$ in $X$. A function $g: \tilde{X} \rightarrow \mathbf{R}$ is continuous if and only if $g \circ q: X \rightarrow \mathbf{R}$ is continuous. From this criterion it follows that if $f: X \rightarrow \mathbf{R}$ is a continuous function which is constant on the classes of equivalences, then the function $g: \tilde{X} \rightarrow \mathbf{R}$ with $g([x])=f(x)$ is well-defined and continuous. 
Recall that a function $u: X \rightarrow \mathbf{R} \cup\{\infty\}$ is called lower semicontinuous (l.s.c.) if at any point $x \in X$

$$
\liminf _{x_{\alpha} \rightarrow x} u\left(x_{\alpha}\right) \geq u(x) .
$$

Equivalently, $u$ is l.s.c. if all lower level sets $\{x \in X: u(x) \leq c\}$ are closed, see [1]. A function $g: \tilde{X} \rightarrow \mathbf{R}$ is l.s.c. if and only if $g \circ q: X \rightarrow \mathbf{R}$ is l.s.c. Indeed, the set $\{[x] \in \tilde{X}: g([x])>c\}$ is open if and only if its inverse image $\{x \in X: g(q(x))>c\}$ is open. If a function $f: X \rightarrow \mathbf{R}$ is l.s.c. and constant on the classes of equivalences, then the function $g: \tilde{X} \rightarrow \mathbf{R}$ with $g([x])=f(x)$ is l.s.c.

A function $u$ is called upper semi-continuous (u.s.c.) if the function $-u$ is lower semicontinuous.

The following result is a generalization of Theorem 2.4 in [8].

Theorem 2.4. Let $\succeq$ be a partial order in a topological space $X$ represented by a countable family $\mathcal{U}=\left\{u_{j}: j=1,2, \ldots\right\}$ of lower semicontinuous functions such that all order intervals $[x, y], y \succeq x$, are compacts. If a subset $\Gamma$ is bounded from above, i.e. $\bar{x} \succeq \Gamma$ for some $\bar{x}$, then Sup $\Gamma$ exists.

Proof. Fix $x_{0} \in \Gamma$. Assuming without loss of generality that $\left|u_{j}\right| \leq 1$ for all $u_{j} \in \mathcal{U}$ we define the function $u:=\sum_{j} 2^{-j} u_{j}$. Then $u$ is l.s.c. and, therefore, for every $x \succeq \Gamma$ it attains its minimum on the non-empty compact set $[\Gamma, x]$. Put

$$
\Lambda(x):=\operatorname{argmin}_{y \in[\Gamma, x]} u(y) .
$$

Then the set $\hat{\Gamma}:=\bigcup_{x \succeq \Gamma} \Lambda(x)$ obviously satisfies the properties $\left(a_{0}\right)$ and $\left(b_{0}\right)$. Let $\hat{x}_{1}, \hat{x}_{2} \in \hat{\Gamma}, \hat{x}_{1} \succeq \hat{x}_{2}$, i.e. $u_{j}\left(\hat{x}_{1}\right) \geq u_{j}\left(\hat{x}_{2}\right)$ for all $j$ and $u\left(\hat{x}_{1}\right) \geq u\left(\hat{x}_{2}\right)$. There is $x_{1} \succeq \Gamma$ such that $\hat{x}_{1} \in \Lambda\left(x_{1}\right)$. Since $\hat{x}_{2} \in\left[\Gamma, \hat{x}_{1}\right] \subseteq\left[\Gamma, x_{1}\right]$, we have that $u\left(\hat{x}_{2}\right) \geq u\left(\hat{x}_{1}\right)$. So, $u\left(\hat{x}_{2}\right)=u\left(\hat{x}_{1}\right)$ and this is possible only if $u_{j}\left(\hat{x}_{1}\right)=u_{j}\left(\hat{x}_{2}\right)$ for all $j$. Therefore, $\hat{x}_{1}=\hat{x}_{2}$ and $\left(c_{0}\right)$ holds.

Remark 2.5. The claim of the above theorem holds also under the assumption of the compactness of the sets $[\Gamma, x]$ for all $x \succeq \Gamma$.

In virtue of the above discussion we have the following generalization.

Theorem 2.6. Let $\succeq$ be a preference relation in $X$ represented by a countable family of lower semicontinuous functions such that all order intervals $[[x],[y]]$, $[y] \succeq[x]$, are compact subsets of $\tilde{X}$. If the subset $\Gamma$ is such that $\bar{x} \succeq \Gamma$ for some $\bar{x}$, then $\operatorname{Sup} \Gamma \neq \emptyset$. 
Proof. The partial order in $\tilde{X}$ is given by the family of lower semicontinuous functions $\{u(f()):. u \in \mathcal{U}\}$ where $f: \tilde{X} \rightarrow X$ is an arbitrary function associating with $[x] \in \tilde{x}$ a point $f(x) \in[x]$. By virtue of Theorem $2.4 \operatorname{Sup} q(\Gamma)$ is not empty and so is the set $\operatorname{Sup} \Gamma=q^{-1}(\operatorname{Sup} q(\Gamma))$.

Remark 2.7. One can observe that in the deterministic setting results involving Sup for a preference relation can be easily obtained, in a standard way, from the corresponding results for partial order in the quotient space.

We finish this subsection by a result showing that on a reasonable level of generality the existence of a continuous multi-utility representation implies the existence of a countable continuous multi-utility representation.

Proposition 2.8. Let $X$ be a $\sigma$-compact metric space. Suppose that a family $\mathcal{U}$ of continuous functions defines a preference relation on $X$. Then this preference relation can be defined by a countable subfamily of $\mathcal{U}$.

Proof. Let $X=\cup_{n} X_{n}$ where $X_{n}$ are compact metric spaces. The metric space $C\left(X_{n}\right)$ of continuous functions is separable and so its subspace $\left.\mathcal{U}\right|_{X_{n}}$ formed by restrictions of functions from $\mathcal{U}$ onto $X_{n}$. Thus, there exists a countable family $\mathcal{U}_{n} \subset \mathcal{U}$ such that the restriction of its elements onto $X_{n}$ are dense in $\left.\mathcal{U}\right|_{X_{n}}$. It is clear that the countable family $\cup_{n} \mathcal{U}_{n}$ defines the same preference relations as $\mathcal{U}$.

Combining this proposition with Theorem 1 in [7] we obtain the following:

Corollary 2.9. Any continuous preference relation on a locally compact and o-compact Hausdorff topological space (in particular, on $\mathbf{R}^{d}$ ) admits a countable multi-utility representation.

In the economic literature compactness assumptions sometimes are considered as too restrictive. In a relation with our hypothesis it may be of interest the following result ([7], Th.3): on a topological space with a countable base any near-complete upper (lower) semicontinuous preorder admits upper (lower) semicontinuous multi-utility representation.

The example below shows that the partial order may not admit a countable multi-utility representation but, nevertheless, any bounded from above subset $\Gamma$ possesses nonempty Sup $\Gamma$.

Example 2.10. Let us consider the space $X=[0,1] \times[0,1] \subset \mathbf{R}^{2}$ with the lexicographic partial order: $(x, y) \succeq\left(x^{\prime}, y^{\prime}\right)$ if either $x>x^{\prime}$ or $x=x^{\prime}$ and $y \geq$ 
$y^{\prime}$. This partial order does not admit a countable multi-utility representation $\left\{u_{j}\right\}$. Indeed, suppose that such a representation exists. Assuming without loss of generality that $0 \leq u_{j} \leq 1$ we define the function $u: X \rightarrow[0,1]$ with $u(x, y)=\sum 2^{-j} u_{j}(x, y)$. Associate with $u$ the set-valued mapping $x \mapsto$ $[u(x, 0), u(x, 1)]$ defined on $[0,1]$. From the definition of the partial order it follows that $u(x, 0)<u(x, 1)$ and $u(x, 1)<u\left(x^{\prime}, 0\right)$ when $x<x^{\prime}$. We get a contradiction since the interval $[0,1]$ cannot contain uncountably many disjoint intervals with non-empty interiors.

Let $\Gamma$ be a non-empty subset of $X$ such that $(x, y) \preceq(\bar{x}, \bar{y})$ for any $(x, y) \in \Gamma$. Let $\hat{x}=\sup \{x:(x, y) \in \Gamma\}$. If there exists $y \in[0,1]$ such that $(\hat{x}, y) \in \Gamma$, then $\operatorname{Sup} \Gamma=\{(\hat{x}, \hat{y})\}$ where $\hat{y}=\sup \{y:(\hat{x}, y) \in \Gamma\}$. If such $y$ does not exist, then $\operatorname{Sup} \Gamma=\{(\hat{x}, 0)\}$.

Note also that for $X=\mathbf{R}^{2}$ the subset $\Gamma=\left\{(x, y) \in \mathbf{R}^{2}: x=1\right\}$ is bounded with respect to the lexicographic partial order but $\operatorname{Sup} \Gamma=\emptyset$.

Example 2.11. Let $X$ be a linear topological space and let $l: X \rightarrow \mathbf{R}$ be a continuous function such that $l(0)=0$ and the inequalities $l(x) \geq 0$ and $l(y) \geq 0$ implies the inequality $l(x+y) \geq 0$. Let $x \succeq y$ means that $l(x-y) \geq 0$. Then $\succeq$ is a continuous preference relations which is a partial order if the system of inequalities $l(x) \geq 0, l(-x) \geq 0$ has only zero solution. Accordingly to Corollary 2.9 this preference relation in $\mathbf{R}^{d}$ admits a countable multiutility representation. In the model of the market with constant proportional transaction costs with $d$ assets where the first one is chosen as the numéraire, the above properties are satisfied by the liquidation function

$$
l(x)=\sup \left\{\lambda: x-\lambda e_{1} \in K\right\}
$$

where $K \subset \mathbf{R}^{d}$ is the solvency cone, [2].

\subsection{Preference Relation in a Hilbert Space Defined by a Cone}

In this subsection we are interested in the preference relation in a (separable) Hilbert space $X$ defined by a closed (convex) cone $G \subseteq X$. As usual, $x \succeq 0$ means that $x \in G$, and $y \succeq x$ means that $y-x \succeq 0$, i.e. $y \in x+G$. Obviously, this preference relation is homogeneous: $y \succeq v$ implies that $\lambda y \succeq \lambda x$ for any $\lambda \geq 0$. Also if $y \succeq x, v \succeq u$, then $x+v \succeq y+u$. It is continuous since its graph $\{(x, y): y-x \in G\}$ is a closed subset of $X \times X$. The preference relation defined by a cone $G$ is a partial order if and only if $G$ is proper, i.e. if $G^{0}=\{0\}$ where $G^{0}:=G \cap(-G)$. 
By the classical separation theorem the cone $G$ is the intersection of the family of closed half-spaces $L=\{x \in X: l x \geq 0\}$ containing $G$. Its complement $G^{c}$ is the union of the open half-spaces $L^{c}$. Notice that in the Hilbert space $X$ any open covering of an open set contains a countable subcovering. Hence, there exists a countable family of vectors $l_{j}$ such that $G=\cap_{j}\left\{x \in X: l_{j} x \geq 0\right\}$. It follows that a countable family of linear functions $u_{j}(x)=l_{j} x$ represents the preference relation defined by $G$. So, the preference relation defined by a closed convex cone $G \subseteq X$ can be generated by a countable family of linear functions. Clearly, the converse is true.

For the preference relation given by a cone the properties defining the set $\hat{\Gamma}=\operatorname{Sup} \Gamma$ can be reformulated in geometric terms as follows:

$\left(a_{0}^{\prime}\right) \hat{\Gamma}-\Gamma \subseteq G$

$\left(b_{0}^{\prime}\right)$ if $x-\Gamma \subseteq G$, then there is $\hat{x} \in \hat{\Gamma}$ such that $x-\hat{x} \in G$;

$\left(c_{0}^{\prime}\right)$ if $\hat{x}_{1}, \hat{x}_{2} \in \hat{\Gamma}$, then $\hat{x}_{1}-\hat{x}_{2} \notin G \backslash G^{0}$.

Notation. To distinguish preference relations generated by various cones (a typical situation in applications for market models with transaction costs) we shall use sometimes the notation $\succeq_{G}$. Note also that the order intervals are convex.

Lemma 2.12. For the partial order defined by a closed proper cone $G$ in $\mathbf{R}^{d}$ the order intervals $[x, y]$ are bounded.

Proof. Suppose that $z_{n} \in[x, y]$ and $\left|z_{n}\right| \rightarrow \infty$. Passing to a subsequence, we may assume without loss of generality that $z_{n} /\left|z_{n}\right| \rightarrow z_{\infty}$ with $\left|z_{\infty}\right|=1$. For any linear function $u(x)$ from the generating family $\mathcal{U}$ the inequalities $u(x) \leq u\left(z_{n}\right) \leq u(y)$ imply that

$$
\frac{u(x)}{\left|z_{n}\right|} \leq u\left(\frac{z_{n}}{\left|z_{n}\right|}\right) \leq \frac{u(y)}{\left|z_{n}\right|} .
$$

It follows that $u\left(z_{\infty}\right)=0$ for all $u \in \mathcal{U}$. That is $z_{\infty}=0$. A contradiction.

Thus, for the case of the partial order on $\mathbf{R}^{d}$ given by a cone the hypotheses of Theorem 2.4 are fulfilled. It is also clear that the arguments in the proof of the above lemma does not work for infinite-dimensional Hilbert space: though one can always find a weakly convergent subsequence of $z_{n} /\left|z_{n}\right|$, the norm of the limit might be well equal to zero. But the convexity of the order intervals combined with the property that the balls in a Hilbert space are weakly compact leads to the following result. 
Theorem 2.13. Let $\succeq$ be the partial order generated by a proper closed convex cone $G$ in a Hilbert space $X$. If $\Gamma \subseteq X$ is such that $\bar{x} \succeq \Gamma$ (i.e. $\bar{x}-\Gamma \subseteq G$ ) for some $\bar{x} \in X$ and for every $x \succeq \Gamma$ the order interval $[\Gamma, x]$ is bounded, then $\operatorname{Sup} \Gamma \neq \emptyset$.

Proof. Take arbitrary $x_{0} \in \Gamma$ and for each $x \succeq \Gamma$ define on the Hilbert space generated by the order interval $[\Gamma, x]$ the linear function $u(. ; x)$ by putting $u(y ; x):=\sum_{j} a_{j}(x) l_{j} y$, where

$$
a_{j}(x):=2^{-j}\left(1+\left|l_{j} x_{0}\right|+\left|l_{j} \bar{x}\right|\right)^{-1} .
$$

The convex closed bounded set $[\Gamma, x]$ is weakly compact. It follows that

$$
\Lambda(x):=\operatorname{argmin}_{y \in[\Gamma, x]} u(y ; x) \neq \emptyset .
$$

In the same way as in the proof of Theorem 2.4 we check that $\hat{\Gamma}:=\bigcup_{x \succeq \Gamma} \Lambda(x)$ satisfies all properties required from $\operatorname{Sup} \Gamma$.

Theorem 2.14. Let $\succeq$ be the preference relation generated by a closed convex cone $G$ in a Hilbert space $X$ and let $q$ be the projection of $X$ onto $\left(G^{0}\right)^{\perp}$. If $\Gamma \subseteq X$ is such that $\bar{x} \succeq \Gamma$ for some $\bar{x} \in X$ and the order intervals $[q(\Gamma), q(x)]$, $x \succeq \Gamma$, are bounded, then $\operatorname{Sup} \Gamma \neq \emptyset$.

Proof. The quotient space $\tilde{X}=X / \sim$ can be identified with $\left(G^{0}\right)^{\perp}$ and the projection $q$ with the quotient mapping. The assumptions ensure that in the quotient space with the induced partial order $\operatorname{Sup} q(\Gamma)$ is non-empty and so is $\operatorname{Sup} \Gamma=q^{-1}(\operatorname{Sup} q(\Gamma))$.

\subsection{Maximum as a Set}

We give definitions of other two sets which are reduced, in the case of usual total order of $\mathbf{R}$ and a bounded subset $\Gamma \in \mathbf{R}$, to the singleton $\{\sup \Gamma\}$. So, they also can be considered as generalizations of the classical notion.

In the sequel $X$ is a topological space with a preference relation on it.

Definition 2.15. Let $\Gamma$ be a non-empty subset of $X$. We put

$$
\operatorname{Max} \Gamma=\{x \in \bar{\Gamma}: \bar{\Gamma} \cap[x, \infty[=[x, x]\} .
$$

Note that $[x, x]=[x]=\{y: y \sim x\}$. In the case of partial order $[x, x]=\{x\}$. 
Definition 2.16. Let $\Gamma$ be a non-empty subset of $X$. We denote by $\operatorname{Max}_{1} \Gamma$ the maximal subset $\hat{\Gamma} \subseteq \bar{\Gamma}$ (possibly empty) such that the following conditions hold:

( $\alpha)$ if $x \in \bar{\Gamma}$, then there is $\hat{x} \in \hat{\Gamma}$ such that $\hat{x} \succeq x$;

( $\beta)$ if $\hat{x}_{1}, \hat{x}_{2} \in \hat{\Gamma}$, then $\hat{x}_{1} \succeq \hat{x}_{2}$ implies $\hat{x}_{1} \sim \hat{x}_{2}$.

Clearly,

$$
\operatorname{Max}_{1} \Gamma \subseteq \operatorname{Max} \Gamma=\operatorname{Max} \bar{\Gamma}=(\operatorname{Max} \Gamma)^{\sim} \cap \bar{\Gamma}=q^{-1}(q(\operatorname{Max} \Gamma)) \cap \bar{\Gamma} .
$$

Similarly,

$$
\operatorname{Max}_{1} \Gamma=\operatorname{Max}_{1} \bar{\Gamma}=\left(\operatorname{Max}_{1} \Gamma\right)^{\sim} \cap \bar{\Gamma}=q^{-1}\left(q\left(\operatorname{Max}_{1} \Gamma\right)\right) \cap \bar{\Gamma} .
$$

Though the use of the word "maximum" seems to be more appropriate in the case of the closed set (and even might confuse some readers when $\Gamma$ is not closed), the adopted notations do not lead to a contradiction in the scalar case. E.g., the open interval $\Gamma=] a, b[\subset \mathbf{R}$ do not have the maximal point but the set Max $\Gamma$ is well-defined: it is the singleton $\{b\}$.

Lemma 2.17. Let $\succeq$ be a preference relation on $X$. Let $\Gamma$ be a non-empty subset of $X$ such that $\overline{q(\Gamma)}=q(\bar{\Gamma})$. Then $q(\operatorname{Max} \Gamma)=\operatorname{Max} q(\Gamma)$.

Proof. Let $[x] \in q(\operatorname{Max} \Gamma)$. Without loss of generality we may assume that the representative $x \in \operatorname{Max} \Gamma$. It follows that $x \in \bar{\Gamma}$ and $[x] \in \overline{q(\Gamma)}$. Suppose that $[y] \in \overline{q(\Gamma)} \cap[[x], \infty[$. By assumption, $\overline{q(\Gamma)}=q(\bar{\Gamma})$ and we may assume that $y \in \bar{\Gamma} \cap[x, \infty[$. Since $x \in \operatorname{Max} \Gamma$, this implies that $y \sim x$, i.e. $[y]=[x]$. Therefore, $q(\operatorname{Max} \Gamma) \subseteq \operatorname{Max} q(\Gamma)$. Of course, the inclusion holds if $\operatorname{Max} \Gamma=\emptyset$. To prove the inverse inclusion, we take $[x] \in \operatorname{Max} q(\Gamma)$. Then $[x] \in q(\bar{\Gamma})$ and we may assume that the representative $x \in \bar{\Gamma}$. If $y \in \bar{\Gamma} \cap[x, \infty[$, then $[y] \in \overline{q(\Gamma)} \cap[[x], \infty[$. Hence $[y]=[x]$, i.e. $y \in[x]$. But this means that $x \in \operatorname{Max} \Gamma$ and $[x] \in q(\operatorname{Max} \Gamma)$. So, $\operatorname{Max} q(\Gamma) \subseteq q(\operatorname{Max} \Gamma)$.

Lemma 2.18. Let $\succeq$ be a preference relation on $X$. Let $\Gamma$ be a non-empty subset of $X$ such that $\overline{q(\Gamma)}=q(\bar{\Gamma})$. Then $q\left(\operatorname{Max}_{1} \Gamma\right)=\operatorname{Max}_{1} q(\Gamma)$.

Proof. Suppose that $\operatorname{Max}_{1} \Gamma \neq \emptyset$. It is easy to check that $q\left(\operatorname{Max}_{1} \Gamma\right)$ satisfies the condition $(\alpha)$ and $(\beta)$ for $\overline{q(\Gamma)}$. Indeed, let $[x] \in \overline{q(\Gamma)}=q(\bar{\Gamma})$. We may assume without loss of generality that $x \in \Gamma$ and, therefore, there exists $y \in \operatorname{Max}_{1} \Gamma$ such that $y \preceq x$. Hence, $[y]=q(y) \preceq[x]$, i.e. $(\alpha)$ holds. In 
the same way we establish $(\beta)$. So, $q\left(\operatorname{Max}_{1} \Gamma\right) \subseteq \operatorname{Max}_{1} q(\Gamma)$. If $\operatorname{Max}_{1} \Gamma=\emptyset$, the inclusion is trivial. If $[x] \in \operatorname{Max}_{1} q(\Gamma)$, we may assume that $x \in \bar{\Gamma}$. It follows that $x \in q^{-1}\left(\operatorname{Max}_{1} q(\Gamma)\right) \cap \bar{\Gamma}$. Hence $x \in \operatorname{Max}_{1} \Gamma$ and $[x] \in q\left(\operatorname{Max}_{1} \Gamma\right)$. That is, $\operatorname{Max}_{1} q(\Gamma) \subseteq q\left(\operatorname{Max}_{1} \Gamma\right)$. If $\operatorname{Max}_{1} q(\Gamma)=\emptyset$, the inclusion is trivial.

The following assertion shows that in the cases important from the point of view of applications both definitions lead to the same subset.

Proposition 2.19. Let $\succeq$ be a partial order represented by a countable family of upper semicontinuous functions and such that all order intervals $[x, y]$, $y \succeq x$, are compacts. Suppose that $\Gamma$ is non-empty and there exists $\bar{x}$ such that $\bar{x} \succeq \Gamma$. Then $\operatorname{Max} \Gamma$ and $\operatorname{Max}_{1} \Gamma$ are non-empty sets and $\operatorname{Max} \Gamma=\operatorname{Max}_{1} \Gamma$.

Proof. The property $(\beta)$ for the set Max $\Gamma$ holds obviously. To check $(\alpha)$ we take a point $x \in \bar{\Gamma}$ and consider the closed set $[x, \infty[\cap \bar{\Gamma}$. Without loss of generality we may assume that $\left|u_{j}\right| \leq 1$. Let us define the upper semi-continuous function $u=\sum 2^{-j} u_{j}$ and put $c:=\sup _{y \in[x, \infty[\cap \bar{\Gamma}} u(y)$. Since $\left[x, \infty[\cap \bar{\Gamma}\right.$ is compact (as a subset of $[x, \bar{x}])$, we obtain that $c=u\left(y_{\infty}\right)$ where $y_{\infty} \in\left[x, \infty\left[\cap \bar{\Gamma}\right.\right.$. Moreover, $\sup _{y \in\left[y_{\infty}, \infty[\cap \bar{\Gamma}\right.} u(y)=u\left(y_{\infty}\right)$ implying that $\left[y_{\infty}, \infty\left[\cap \bar{\Gamma}=\left\{y_{\infty}\right\}\right.\right.$. Therefore, $y_{\infty} \in \operatorname{Max} \Gamma$ and $(\alpha)$ holds. The claim follows now from the uniqueness of $\operatorname{Max}_{1} \Gamma$.

The generalization to the preference relation by the passage to the quotient space is not straightforward because the image of the closed set under a continuous mapping, even such a simple one as a projection in $\mathbf{R}^{d}$, in general, may not be closed. Nevertheless, with the help of the above lemmata we easily deduce from Proposition 2.19 the following:

Corollary 2.20. Let $\succeq$ be a preference relation represented by a countable family of upper semicontinuous functions and such that all order intervals $[q(x), q(y)], y \succeq x$, are compacts. Suppose that there exists $\bar{x}$ such that $\bar{x} \succeq \Gamma$ and, moreover, $\overline{q(\Gamma)}=q(\bar{\Gamma})$. Then $\operatorname{Max} \Gamma$ and $\operatorname{Max}_{1} \Gamma$ are non-empty sets and $\operatorname{Max} \Gamma=\operatorname{Max}_{1} \Gamma$.

Note that in the standard model of a market with transaction costs where $\succeq$ is the preference relation in $\mathbf{R}^{d}$ defined by the solvency cone the condition on $\Gamma$ holds when $\Gamma$ is a polyhedron or a polyhedral cone: these classes of sets are stable under linear mappings.

Remark 2.21. The hypotheses of Proposition 2.19 on the partial order is fulfilled if the latter is generated by a closed convex proper cone in $\mathbf{R}^{d}$ (Proposition 2.6 in [8]). For a general partial order we cannot claim that the sets 
$\operatorname{Max} \Gamma$ and $\operatorname{Max}_{1} \Gamma$ coincide. Indeed, let us consider the partial order in $\mathbf{R}^{2}$ given by the set

$$
G:=\{(0,0)\} \cup\left\{(x, y) \in \mathbf{Z}_{+}^{2}: x+y \geq 2\right\} .
$$

Then for $\Gamma=\{(-1,-1)\} \cup\left\{(i, i): i \in \mathbf{Z}_{+}\right\}$we have $\operatorname{Sup} \Gamma=\operatorname{Max}_{1} \Gamma=\emptyset$ while $\operatorname{Max} \Gamma=\{(-1,-1)\}$.

Remark 2.22. In the book [11] one can find a definition of supremum useful in the vector optimization theory. For the case of $\mathbf{R}^{d}$ with the partial order given by a convex cone $G \neq \mathbf{R}^{d}$ with non-empty interior it is given as follows. First, one defines the lower closure of $A$ as the set

$$
\mathrm{Cl}_{-} A:=\left\{x \in \mathbf{R}^{d}: x-\operatorname{int} G \subseteq A-\operatorname{int} G\right\}
$$

and the set of weak maximal points of $A$

$$
\text { wMax } A:=\{x \in A:(x+\operatorname{int} G) \cap A=\emptyset\} .
$$

Finally, $\operatorname{Sup}_{w} A:=\operatorname{wMax}_{\mathrm{Cl}} A$. For the cone $G=\mathbf{R}_{+}^{d}$ and $A=-\mathbf{R}^{d}$ we have the equalities $\mathrm{Cl}_{-} A=A$ and $\operatorname{Sup}_{w} A=\operatorname{wMax} A=-\partial \mathbf{R}_{+}^{d}$. According to our definitions $\operatorname{Sup} A=\operatorname{Max} A=\operatorname{Max}_{1} A=\{0\}$.

Remark 2.23. Let $X=\mathbf{R}^{2}$, the partial order is generated by the cone $\mathbf{R}_{+}^{2}$. Let $\Gamma$ be the unit disc $\{x:|x| \leq 1\}$. Then $\operatorname{Sup} \Gamma=(1,1)$ while the set $\operatorname{Max} \Gamma=\Gamma \cap \mathbf{R}_{+}^{2}$.

Remark 2.24. The reader may ask about the correspondence of the introduced concepts with those of the multicriteria optimization. Of course, such relations do exist and merit to be understood. For simplicity, let us consider the simplest Pareto maximization problem in $\mathbf{R}^{d}$ :

$$
u(x) \rightarrow \max , \quad x \in \Gamma,
$$

where $u: \mathbf{R}^{d} \rightarrow \mathbf{R}^{n}$ is the objective function taking values in the Euclidean space equipped with the partial order $\geq$ defined by the cone $\mathbf{R}_{+}^{n}$, and $\Gamma$ is a closed subset of $\mathbf{R}^{d}$. We denote by $\succeq$ the preference relation in $\mathbf{R}^{d}$ induced by $u$, i.e. having the family $\left\{u^{j}(),. 1 \leq j \leq n\right\}$ as the multi-utility representation. In the terminology of the book [6] (see p. 24, Definition 2.1) the point $\hat{x} \in \Gamma$ is called efficient or Pareto optimal if there is no other $x \in \Gamma$ such that $u(x) \geq u(\hat{x})$. Let $\Gamma_{E}$ denote the set of all efficient points. Suppose that $\succeq$ is a partial order. It is easily seen that the efficient set $\Gamma_{E}$ coincides with $\operatorname{Max} \Gamma$. An extension of this example to the case of countable set of criteria is obvious. 


\section{Essential Supremum and Essential Maximum in $L^{0}(X, \mathcal{F})$}

\subsection{Essential Supremum in a General Setting}

Let $X$ be a separable metric space with its Borel $\sigma$-algebra $\mathcal{B}_{X}$ and let $(\Omega, \mathcal{F}, P)$ be a probability space. Let $\mathcal{H}$ be a sub- $\sigma$-algebra of $\mathcal{F}$. We consider in the space $L^{0}(X, \mathcal{F})$ (of classes of equivalence) of $X$-valued random variables a preference relation defined by a countable family $\mathcal{U}=\left\{u_{j}: j=1,2, \ldots\right\}$ of Carathéodory functions $u_{j}: \Omega \times X \rightarrow \mathbf{R}$, i.e. functions with the following properties:

(i) $u_{j}(., x) \in L^{0}(\mathbf{R}, \mathcal{F})$ for every $x \in X$;

(ii) $u_{j}(\omega,$.$) is continuous for almost all \omega \in \Omega$.

Namely, for elements $\gamma_{1}, \gamma_{2} \in L^{0}(X, \mathcal{F})$, the relation $\gamma_{2} \succeq \gamma_{1}$ means that $u_{j}\left(\gamma_{2}\right) \succeq u_{j}\left(\gamma_{1}\right)$ (a.s.) for all $j$. Recall that the Carathéodory functions are jointly measurable and so the superpositions $u_{j}\left(\gamma_{1}\right), u_{2}\left(\gamma_{1}\right)$ are random variables. The equivalence relation $\gamma_{2} \sim \gamma_{1}$ has an obvious meaning.

Note that for every $\omega$ (except a null set) the countable family of functions $\left\{u_{j}(\omega,).\right\}$ defines a preference relation in $X$. Abusing language, we refer to $\mathcal{U}$ as to the family representing a partial order in $L^{0}(X, \mathcal{F})$. In the sequel we associate with an order interval $\left[\gamma_{1}, \gamma_{2}\right]$ in $L^{0}(X, \mathcal{F})$ the order intervals $\left[\gamma_{1}(\omega), \gamma_{2}(\omega)\right]$ in $X$ corresponding to these families.

Definition 3.1. Let $\Gamma$ be a subset of $L^{0}(X, \mathcal{F})$. We denote by $\mathcal{H}$-Esssup $\Gamma$ the maximal subset $\hat{\Gamma}$ of $L^{0}(X, \mathcal{H})$ such that the following conditions hold:

(a) $\hat{\Gamma} \succeq \Gamma$;

(b) if $\gamma \in L^{0}(X, \mathcal{H})$ and $\gamma \succeq \Gamma$, then there is $\hat{\gamma} \in \hat{\Gamma}$ such that $\gamma \succeq \hat{\gamma}$;

(c) if $\hat{\gamma}_{1}, \hat{\gamma}_{2} \in \hat{\Gamma}$, then $\hat{\gamma}_{1} \succeq \hat{\gamma}_{2}$ implies $\hat{\gamma}_{1} \sim \hat{\gamma}_{2}$.

If $\mathcal{H}$-Esssup $\Gamma$ is a singleton, we denote by $\mathcal{H}$-esssup $\Gamma$ its unique element.

When needed, we denote $\operatorname{Esssup}^{\mathcal{U}} \Gamma$ where $\mathcal{U}$ is the family of functions representing the preference relation.

Inspection of the proof of Theorem 3.7 in [8] (which deals with a partial order in $\mathbf{R}^{d}$ ) allows us to formulate the following statement:

Theorem 3.2. Let $\succeq$ be a preference relation in $L^{0}(X, \mathcal{F})$ represented by a countable family of Carathéodory functions, i.e. satisfying (i), (ii), such that $\left|u_{j}\right| \leq 1$. Let $\Gamma \neq \emptyset$ be such that $\bar{\gamma} \succeq \Gamma$ for some $\bar{\gamma} \in L^{0}(X, \mathcal{H})$. Suppose that for any $\gamma \in L^{0}([\Gamma, \infty[, \mathcal{H})$

$$
\Lambda(\gamma)=\operatorname{argmin}_{\zeta \in L^{0}([\Gamma, \gamma], \mathcal{H})} E u(\zeta) \neq \emptyset,
$$


where $u(\omega, z)=\sum 2^{-j} u_{j}(\omega, z)$. Then

$$
\mathcal{H} \text {-Esssup } \Gamma=\cup_{\gamma \in L^{0}([\Gamma, \infty], \mathcal{H})} \Lambda(\gamma) \neq \emptyset .
$$

Clearly, this formulation is too technical to be considered as a satisfactory result. The main part of the proof Theorem 3.7 in [8] consists in checking that the condition (3.1) is fulfilled under hypothesis that the $\omega$-sections of the order intervals $\left[\gamma_{1}, \gamma_{2}\right]$ are compact. The arguments used heavily that the space is finite-dimensional. In Section 4 we investigate the case where $X$ is a Hilbert space and the preference relation are given by a random cone in it.

\subsection{Essential Maximum}

First, we recall some classical concepts, see, e.g. [13], [9].

The set $\Gamma \subseteq L^{0}(X, \mathcal{F})$ is $\mathcal{H}$-decomposable if for any $\gamma_{1}, \gamma_{2} \in \Gamma$ and $A \in \mathcal{H}$ the random variable $\gamma_{1} I_{A}+\gamma_{2} I_{A^{c}} \in \Gamma$.

We denote by $\operatorname{env}_{\mathcal{H}} \Gamma$ the smallest $\mathcal{H}$-decomposable subset of $L^{0}(X, \mathcal{F})$ containing $\Gamma$ and by $\operatorname{clenv}_{\mathcal{H}} \Gamma$ its closure in $L^{0}(X, \mathcal{F})$.

It is easily seen that $\operatorname{env}_{\mathcal{H}} \Gamma$ is the set of all random variables of the form $\sum \gamma_{i} I_{A_{i}}$ where $\gamma_{i} \in \Gamma$ and $\left\{A_{i}\right\}$ is an arbitrary finite partition of $\Omega$ into $\mathcal{H}$ measurable subsets. It follows from this alternative description that the set $\mathcal{H}$-cl env $\Gamma$ is $\mathcal{H}$-decomposable.

Definition 3.3. Let $\Gamma$ be a non-empty subset of $L^{0}(X, \mathcal{F})$. We put

$$
\mathcal{H} \text {-Essmax } \Gamma=\left\{\gamma \in \operatorname{clenv}_{\mathcal{H}} \Gamma: \operatorname{clenv}_{\mathcal{H}} \Gamma \cap[\gamma, \infty[=[\gamma, \gamma]\}\right.
$$

Definition 3.4. Let $\Gamma$ be a non-empty subset of $L^{0}(X, \mathcal{F})$. We denote by $\mathcal{H}$ Essmax $_{1} \Gamma$ the largest subset $\hat{\Gamma} \subseteq \operatorname{cl~env~}_{\mathcal{H}} \Gamma$ such that the following conditions hold:

(i) if $\gamma \in \operatorname{clenv}_{\mathcal{H}} \Gamma$, then there is $\hat{\gamma} \in \hat{\Gamma}$ such that $\hat{\gamma} \succeq \gamma$;

(ii) if $\hat{\gamma}_{1}, \hat{\gamma}_{2} \in \hat{\Gamma}$, then $\hat{\gamma}_{1} \succeq \hat{\gamma}_{2}$ implies $\hat{\gamma}_{1} \sim \hat{\gamma}_{2}$.

The definitions of $\mathcal{H}$-Essmin $\Gamma$ and $\mathcal{H}$-Essmin ${ }_{1} \Gamma$ are obvious.

In the case where $\succeq$ is a partial order we have in Definition $3.3[\gamma, \gamma]=\{\gamma\}$ and in the condition (ii) above the property $\hat{\gamma}_{1} \sim \hat{\gamma}_{2}$ means that $\hat{\gamma}_{1}=\hat{\gamma}_{2}$. Clearly, the set with the above properties is uniquely defined. Though our definitions are given for $\Gamma \in L^{0}(X, \mathcal{F})$, the most important is the case where $\Gamma \in L^{0}(X, \mathcal{H})$. 
Proposition 3.5. Let $\succeq$ be a partial order in $L^{0}\left(\mathbf{R}^{d}, \mathcal{F}\right)$ represented by a countable family of functions satisfying $(i)$, (ii) and such that all order intervals $\left[\gamma_{1}(\omega), \gamma_{2}(\omega)\right], \gamma_{2} \succeq \gamma_{1}$, are compacts a.s. Let $\Gamma$ be a non-empty subset of $L^{0}\left(\mathbf{R}^{d}, \mathcal{H}\right)$. Suppose that there exists $\bar{\gamma} \in L^{0}\left(\mathbf{R}^{d}, \mathcal{H}\right)$ such that $\bar{\gamma} \succeq \Gamma$. Then $\mathcal{H}$-Essmax $\Gamma=\mathcal{H}$-Essmax $\operatorname{Es}_{1} \Gamma \emptyset$.

Proof. The arguments are similar to those of Proposition 2.19. Note that the set $\mathcal{H}$-Essmax $\Gamma$ obviously satisfies $(i i)$ and it remains only to check $(i)$. For $\gamma \in \operatorname{clenv}_{\mathcal{H}} \Gamma$, we put

$$
c:=\sup _{\tilde{\gamma} \in \operatorname{clenv} \mathcal{H} \Gamma \cap L^{0}([\gamma, \infty), \mathcal{H})} E u(\tilde{\gamma}) .
$$

Let $\left(\tilde{\gamma}_{n}\right)$ be a sequence on which the supremum in the above definition is attained. As the set $\operatorname{clenv}_{\mathcal{H}} \Gamma \subseteq L^{0}\left(\mathbf{R}^{d}, \mathcal{H}\right)$ is decomposable, we may assume without loss of generality (by applying Lemma 2.1.2 [9] on convergent subsequences) that the sequence of conditional expectations $E\left(u\left(\gamma_{n}\right) \mid \mathcal{H}\right)$ is increasing and $\tilde{\gamma}_{n}$ converges a.s. to some $\tilde{\gamma}_{\infty} \in \operatorname{clenv}_{\mathcal{H}} \Gamma \cap L^{0}([\gamma, \infty), \mathcal{H})$ such that $c:=E u\left(\tilde{\gamma}_{\infty}\right)$.

By definition of $c$, it is straightforward that $\tilde{\gamma}_{\infty} \in \mathcal{H}$-Essmax $\Gamma$ and the conclusion follows.

Corollary 3.6. Let $\succeq$ be a preference relation in $L^{0}\left(\mathbf{R}^{d}, \mathcal{F}\right)$ defined by a random cone $G$. Let $\Gamma=L^{0}(B, \mathcal{F})$ where $B$ is a measurable set-valued mapping with non-empty closed sections. Suppose that the projections $q(\omega, B(\omega))$ onto $G_{0}^{\perp}(\omega)$ are closed sets a.s. and $\bar{\gamma} \succeq \Gamma$ for some $\bar{\gamma} \in L^{0}\left(\mathbf{R}^{d}, \mathcal{F}\right)$. Then $\mathcal{F}$-Essmax $\Gamma=\mathcal{F}$-Essmax ${ }_{1} \Gamma \neq \emptyset$.

Proof. Slightly abusing the notation, we consider the "lifted" mapping $q: L^{0}\left(\mathbf{R}^{d}, \mathcal{F}\right) \rightarrow L^{0}\left(q\left(\mathbf{R}^{d}\right), \mathcal{F}\right)$, defined in the natural way in the space of (classes of) random variables. It is easy to see that $q(\Gamma)=L^{0}(q(B), \mathcal{F})$. According to the above proposition

$$
\mathcal{F} \text {-Essmax } q(\Gamma)=\mathcal{F} \text {-Essmax } 1 q(\Gamma) \neq \emptyset \text {. }
$$

The claim follows now from (2.1), (2.2) and Lemmata 2.17, 2.18.

\section{Essential Supremum in $L^{0}(X)$ with Respect to a Random Cone}

\subsection{Setting}

Let $(\Omega, \mathcal{F}, P)$ be a complete probability space and let $X$ be a separable Hilbert space. Let $\omega \mapsto G(\omega) \subseteq X$ be a measurable set-valued mapping 
whose values are closed convex cones. The measurability is understood as the measurability of the graph, i.e.

$$
\operatorname{graph} G:=\{(\omega, x) \in \Omega \times X: x \in G(\omega)\} \in \mathcal{F} \otimes \mathcal{B}(X) .
$$

The positive dual $G^{*}$ of $G$ is defined as the measurable mapping whose values are closed convex cones

$$
G^{*}(\omega):=\{x \in X: x y \geq 0, \forall y \in G(\omega)\}
$$

where $x y$ is the scalar product generating the norm $\|$.$\| in X$. Note that $0 \in L^{0}(G, \mathcal{F}) \neq \emptyset$.

Recall that a measurable mapping whose values are closed subsets admits a Castaing representation. In our case this means that there is a countable set of measurable selectors $\xi_{i}$ of $G$ such that $G(\omega)=\overline{\left\{\xi_{i}(\omega): i \in \mathbf{N}\right\}}$ for all $\omega \in \Omega$. Thus,

$$
\operatorname{graph} G^{*}=\left\{(\omega, y) \in \Omega \times X: y \xi_{i}(\omega) \geq 0, \forall i \in \mathbf{N}\right\} \in \mathcal{F} \otimes \mathcal{B}(X),
$$

i.e. $G^{*}$ is a measurable mapping and admits a Castaing representation, i.e. there exists a countable set of $\mathcal{G}$-measurable selectors $\eta_{i}$ of $G^{*}$ such that $G^{*}(\omega)=\overline{\left\{\eta_{i}(\omega): i \in \mathbf{N}\right\}}$ for all $\omega \in \Omega$.

Since $G=\left(G^{*}\right)^{*}$,

$$
G(\omega)=\left\{(\omega, x) \in \Omega \times X: \eta_{i}(\omega) x \geq 0, \forall i \in \mathbf{N}\right\}
$$

The relation $\gamma_{2}-\gamma_{1} \in G$ (a.s.) defines a preference relation $\gamma_{2} \succeq \gamma_{1}$ in $L^{0}(X, \mathcal{F})$. Moreover, the countable family of functions $u_{j}(\omega, x)=\eta_{j}(\omega) x$ where $\eta_{j}$ is a Castaing representation of $G^{*}$, represents the preference relation defined by $G$ which is a partial order when the sections of $G$ are proper cones.

Notation. Let $\mathcal{H}$ be a sub- $\sigma$-algebra of $\mathcal{F}$ and let $\Gamma \subseteq L^{0}(X, \mathcal{F})$. We shall use the notation $(\mathcal{H}, G)$-Esssup $\Gamma$ instead of $\mathcal{H}$-Esssup $\Gamma$ to indicate that partial order is generated by the random cone $G$.

Theorem 4.1. Let $X$ be a separable Hilbert space and let $\succeq$ be a preference relation in $L^{0}(X, \mathcal{F})$ defined by a random cone $G$. Suppose that the subspaces $\left(G^{0}(\omega)\right)^{\perp}$ are finite-dimensional a.s. Let $\Gamma \neq \emptyset$ be such that $\bar{\gamma} \succeq \Gamma$ for some $\bar{\gamma} \in L^{0}(X, \mathcal{F})$. Then $\mathcal{F}$-Esssup $\Gamma \neq \emptyset$. 
Proof. (a) Take arbitrary $\gamma_{0} \in \Gamma$ and for each $\gamma \in L^{0}(X, \mathcal{F}), \gamma \geq \Gamma$, define the random variables

$$
\alpha_{j}(\gamma):=\alpha_{j}(\omega, \gamma):=2^{-j}\left(1+\left|\eta_{j}(\omega) \gamma_{0}(\omega)\right|+\left|\eta_{j}(\omega) \bar{\gamma}(\omega)\right|\right)
$$

Put

$$
u(x, \gamma):=u(x, \omega, \gamma):=\sum_{j} a_{j}(\omega, \gamma) \eta_{j}(\omega) x
$$

Then the mapping $\xi \mapsto u(\xi, \gamma)$ is well-defined for $\xi \in[\Gamma, \gamma]$ and for such an argument $u(\xi, \gamma)$ is a random variable with values in the interval $[-1,1]$. Let

$$
c(\gamma):=\inf _{\xi} E u(\xi, \gamma)
$$

where infimum is taken over all $\xi \in[\Gamma, \gamma] \cap L^{0}(X, \mathcal{F})$. Let $\xi_{n}$ be a minimizing sequence of random variables (not just classes of equivalences). Without loss of generality we may assume that $\xi_{n}(\omega)=q\left(\omega, \xi_{n}(\omega)\right)$ a.s., where $q(\omega,$.$) is a$ projection of $X$ onto $\left(G^{0}(\omega)\right)^{\perp}$. Moreover, we may assume that the sequence $u\left(\xi_{n}, \gamma\right)$ is decreasing almost surely. Let us consider the set

$$
A:=\left\{(\omega, x) \in \Omega \times X: \lim _{n} \inf _{m \geq n}\left\|\xi_{m}(\omega)-x\right\|=0\right\} \in \mathcal{F} \otimes \mathcal{B}(X) .
$$

Let $q(\Gamma):=\{\xi: \xi(\omega)=q(\omega, \gamma(\omega))\}$. Since $\left(G^{0}(\omega)\right)^{\perp}$ are finite-dimensional a.s., the $\omega$-sections of $[q(\Gamma), q(\gamma)]$ are compact a.s. Hence, almost all $\omega$ sections of $A$ not empty. Take an almost sure measurable selector of $A$, i.e. an $X$-valued random variable $\xi$ such that $\lim _{n} \inf _{m \geq n}\left\|\xi_{n}-\xi\right\|=0$ a.s. It is clear that $c(\gamma)=E u(\xi ; \gamma)$. Thus, the sets $\Lambda(\gamma)$ are non-empty and we can conclude in the usual way that the set $\mathcal{F}$-Esssup $\Gamma \neq \emptyset$.

\section{Applications to Models of Financial Markets with Transaction Costs}

In the model we are given a stochastic basis $\left(\Omega, \mathcal{F}, \mathbf{F}=\left(\mathcal{F}_{t}\right)_{t=0, \ldots, T}, P\right)$ with a $d$-dimensional adapted process $S=\left(S_{t}\right)$ with strictly positive components and an adapted set-valued process $K=\left(K_{t}\right)$ whose values are closed convex cones $K_{t} \subset \mathbf{R}^{d}$ with the interiors containing $\mathbf{R}_{+}^{d} \backslash\{0\}$.

Define the random diagonal operators

$$
\phi_{t}:\left(x^{1}, \ldots, x^{d}\right) \mapsto\left(x^{1} / S_{t}^{1}, \ldots, x^{d} / S_{t}^{d}\right), \quad t=0, \ldots, T
$$


and relate with them the random cones $\widehat{K}_{t}:=\phi_{t} K_{t}$. The random cones $K_{t}$ and $\widehat{K}_{t}$, corresponding to accountability in monetary and physical units, respectively, induce preference relations on $L^{0}\left(\mathbf{R}^{d}, \mathcal{F}\right)$ (which are partial orders in the case when the cones are proper, i.e. the efficient friction condition is fulfilled.)

We consider the set $\widehat{\mathcal{V}}$ of $\mathbf{R}^{d}$-valued adapted processes $\widehat{V}$ such that the increments $\Delta \widehat{V}_{t}:=\widehat{V}_{t}-\widehat{V}_{t-1} \in-\widehat{K}_{t}$ for all $t$ and the set $\mathcal{V}$ which elements are the processes $V$ with $V_{t}=\phi_{t}^{-1} \widehat{V}_{t}, \widehat{V} \in \widehat{\mathcal{V}}$.

In the context of the theory of markets with transaction costs, $K_{t}$ are the solvency cone corresponding to the description of the model in terms of a numéraire, $\mathcal{V}$ is the set of value processes of self-financing portfolios. The notations with hat correspond to the description of the model in terms of "physical" units where the portfolio dynamics is much simpler because it does not depend on price movements. A typical example is the model of currency market defined via the adapted matrix-valued process of transaction costs coefficients $\Lambda=\left(\lambda_{t}^{i j}\right)$. In this case

$$
K_{t}=\text { cone }\left\{\left(1+\lambda_{t}^{i j}\right) e_{i}-e_{j}, e_{i}, 1 \leq i, j \leq d\right\} .
$$

In this model European contingent claims are $d$-dimensional random vectors while American contingent claims are adapted $d$-dimensional random processes. In accordance to the notation adopted in [9] we shall use the notation $Y=\left(Y_{t}\right)$ when the American contingent claim is expressed in units of the numéraire and $\widehat{Y}=\left(\widehat{Y}_{t}\right)$ when it is expressed in physical units. The relation is obvious: $\widehat{Y}_{t}=\phi_{t} Y_{t}$.

One of principal problems of practical importance in the theory of financial markets is to find the set of hedging capitals and hedging strategies, starting from the minimal initial capital, and develop numerical algorithms for their implementations, e.g., for multinomial models, see [12] and references therein. Since the hedging strategies, in general, are not unique it seems reasonable to look for hedging strategies with additional properties. This idea was exploited in [8] where it was considered the hedging problem for European contingent claim $\widehat{Y}_{T}$ and for the minimal strategies was obtained a recurrent system of backward inclusions involving the concept of Esssup. In this paper for the problem of hedging of an American option (it is quite different from that of a European one, see [9]) we obtain a recurrent system of backward inclusions involving Essmax 1 . 
The value process $\widehat{V} \in \widehat{\mathcal{V}}$ is called minimal if $\widehat{V} \succeq_{\widehat{K}} \widehat{Y}$ and any process $\widehat{W} \in \widehat{\mathcal{V}}$ such that $\widehat{Y} \preceq_{\widehat{K}} \widehat{W} \preceq_{\widehat{K}} \widehat{V}$ coincides with $\widehat{V}$. The notation means that to compare values of the processes at time $t$ one uses the partial order generated by the random cone $\widehat{K}_{t}$. We denote $\widehat{\mathcal{V}}_{\text {min }}$ the set of all minimal processes.

Proposition 5.1. Suppose there exists a process $\hat{V}^{0} \in \widehat{\mathcal{V}}$ such that $\widehat{V}^{0} \succeq_{\widehat{K}} \widehat{Y}$ Then the set $\mathcal{V}_{\min }$ is non-empty and coincides with the set of solutions of backward inclusions

$\widehat{V}_{t} \in\left(\mathcal{F}_{t}, \widehat{K}_{t}\right)-\operatorname{Essmin}_{1} L^{0}\left(\left(\widehat{Y}_{t}+\widehat{K}_{t}\right) \cap\left(\widehat{V}_{t+1}+\widehat{K}_{t+1}\right), \mathcal{F}_{t}\right), \quad t \leq T-1, \quad \widehat{V}_{T}=\widehat{Y}_{T}$.

Proof. Using Corollary 3.6 (or Theorem 4.1 in the case of efficient friction) and the backward induction we obtain that the set of solutions of the inclusions (5.1) is nonempty and Essmin $=$ Essmin $_{1}$.

Take an arbitrary $\widehat{W} \in \widehat{\mathcal{V}}$ such that $\widehat{W} \succeq_{\widehat{K}} \widehat{Y}, \widehat{W}_{T}=\widehat{Y}_{T}$ and assume that $\widehat{W} \preceq_{\widehat{K}} \widehat{V}$ where $\widehat{V}$ satisfies the relations (5.1). Since $\Delta \widehat{W}_{t+1} \in-\widehat{K}_{t+1}$, we obtain, assuming the equality $\widehat{V}_{t+1}=\widehat{W}_{t+1}$, that

$\widehat{W}_{t} \in L^{0}\left(\left(\widehat{Y}_{t}+\widehat{K}_{t}\right) \cap\left(\widehat{W}_{t+1}+\widehat{K}_{t+1}\right), \mathcal{F}_{t}\right)=L^{0}\left(\left(\widehat{Y}_{t}+\widehat{K}_{t}\right) \cap\left(\widehat{V}_{t+1}+\widehat{K}_{t+1}\right), \mathcal{F}_{t}\right)$.

Using the definition of Essmin 1 we get that $\widehat{V}_{t} \preceq_{\widehat{K}_{t}} \widehat{W}_{t}$, hence $\widehat{V}_{t}=\widehat{W}_{t}$. The backward induction argument leads to the conclusion that $\widehat{V}=\widehat{W}$.

Remark 5.2. Suppose for simplicity that all ordering cones are proper and assume that the model admits a strictly consistent price system. Then the convex set of hedging initial capitals $\widehat{D}$ is closed, see [9], Th.3.3.3. Let $x$ be a minimal point of this set in the sense of the partial order induced by $\widehat{K}_{0}$, i.e. if $y \in \widehat{D}$ and $y \preceq_{\widehat{K}_{0}} x$, then $y=x$. Then there is a value process $\widehat{V} \in \mathcal{V}_{\text {min }}$ such that its initial value $\widehat{V}_{-1}=\widehat{V}_{0}=x$. Indeed, by definition of $\widehat{D}$ there is exists a portfolio process $\widehat{W}$ such that $\widehat{W}_{-1}=x$ and $\widehat{W} \succeq \widehat{Y}$. Accordingly to the above proposition there exists a minimal dominating process $\widehat{V}$ for which we have in particular $\widehat{V}_{0} \preceq x$. Thus, $\widehat{V}_{0}=x$ and we can take the initial value $\widehat{V}_{-1}=x$. On the other hand, any value process $\widehat{V} \in \mathcal{V}_{\text {min }}$ starts from the initial value which is a minimal element of $\widehat{D}$.

Remark 5.3. The extension of the concepts of the present paper to the case of random processes in discrete time seems to be rather straightforward. 
More challenging seems to be the case of random processes in continuous-time which will be explored elsewhere.

[1] Aliprantis Ch., Border K. Infinite Dimensional Analysis: A Hitchhiker's Guide. Springer-Verlag, 2006.

[2] Bouchard B., Kabanov Yu., Touzi N. Option pricing by large risk aversion utility under transaction costs. Decision in Economics and Finance, 24 (2001), 127-136.

[3] Bouchard B., Lépinette E., Taflin E. Robust no-free lunch with vanishing risk, a continuum of assets and proportional transaction costs. Preprint.

[4] Bouchard B., Taflin E. No-arbitrage of second kind in countable markets with proportional transaction costs. Annals of Applied Probability, 23, (2013), 427-454.

[5] Dolecki S., Malivert Ch. General duality in vector optimization. Optimization 27 (1993), 97119.

[6] Ehrgott M. Multicriteria Optimization, 2nd edition. Springer, 2005.

[7] Evren O., Ok E.A. On the multi-utility representation of preference relations. Journal of Mathematical Economics, 14 (2011), 4-5, 554-563.

[8] Kabanov Yu., Lépinette E. Essential supremum with respect to a random partial order. Preprint.

[9] Kabanov Yu., Safarian M. Markets with Transaction Costs. Mathematical Theory. Springer, 2009.

[10] Kelly J. General Topology. Van Nostrand, 1957.

[11] Löhne A. Vector Optimization with Infimum and Supremum. Springer, 2011.

[12] Löhne A., Rudloff B. An algorithm for calculating the set of superhedging portfolios and strategies in markets with transaction costs. Preprint at arXiv, 2012.

[13] Molchanov I. Theory of Random Sets. Springer, 2005. 
[14] Pennanen T., Penner I. Hedging of claims with physical delivery under convex transaction costs. SIAM J. Financial Mathematics, 1 (2010), 158-178.

[15] Tanino T. On supremum of a set in a multi-dimensional space. Journal of Mathematical Analisis and Applications, 130 (1998), 386-397. 